\title{
Becoming "Teletherapeutic": Harnessing Accelerated Experiential Dynamic Psychotherapy (AEDP) for Challenges of the Covid-19 Era
}

\author{
Idit H. Ronen-Setter ${ }^{1}$ (i) Esther Cohen ${ }^{2}$
}

Published online: 15 June 2020

○) Springer Science+Business Media, LLC, part of Springer Nature 2020

\begin{abstract}
The covid-19 pandemic raises substantial challenges for the practice of psychotherapy. The rapid changes in the personal experiences of both clients and therapists, and the required adaptations in the therapeutic setting, affect the therapeutic relationship and its process. We examine common challenges observed through supervision of therapists and peer group discussions, viewing them in light of reports of previous collective traumatic events. Consequently, we introduce major premises and techniques borrowed from Accelerated Experiential Dynamic Psychotherapy as they may apply to teletherapy. These interventions aim to maintain and strengthen the intimacy and safety of the therapeutic attachment relationship, essential for processing actual experiences of emotions and creating affective changes. We explicate the rationale and the clinical application of these relational and experiential interventions, and organize them through a comprehensive model. The model visually illustrates the matching of therapeutic interventions to the handling of the psychological upheavals triggered by the changes imposed by the pandemic, particularly the move to teletherapy. In addition to theoretical and practical suggestions, which could be adapted to various models of therapy, we present a brief clinical case demonstrating the application of the suggested therapeutic thinking and interventions.
\end{abstract}

Keyword Teletherapy · Accelerated experiential dynamic psychotherapy · AEDP · Covid-19 · Coronavirus · 'Shared traumatic reality'

The Covid-19 pandemic presents a unique set of challenges for managing therapeutic processes, requiring an integrative, comprehensive model of therapy suited for these unusual times. In this paper, we map some of these challenges by incorporating previous and recent research related to therapeutic work conducted under conditions of collective traumatic events and disasters derived from our current experiences supervising work with therapists and from ongoing discussion groups of colleagues. Closely observing emerging obstacles related to conditions of government-prescribed home-confinement, social distancing and

Idit H. Ronen-Setter

Idit.setter@gmail.com

Esther Cohen

esther.cohen@mail.huji.ac.il

1 AEDP-Israel branch, The AEDP Institute, New York, USA

2 School of Education, The Hebrew University of Jerusalem, Jerusalem, Israel quarantine, we focus on ideas for meeting the new challenges to psychotherapy.

\section{Psychotherapy-Relevant Experiential Changes Associated with Covid-19}

During individual supervision and peer-group meetings, we identified four distinctive spheres that characterize the main challenges that Covid-19 presents to psychotherapy. These include: the client's experiences of change associated with the pandemic, the therapist's parallel experiences of change, ensuing changes in the therapeutic relationship, and the required transition to teletherapy.

\section{Client's Experiences of Change}

Recent reviews of the psychological implications of the Covid-19 pandemic for the general population show an increase in psychological distress and risk for negative mental health consequences (Asmundson and Taylor 2020; 
Brooks et al. 2020). These may comprise fears of infection and health anxiety; feelings of guilt for contributing to infection; fear of personal financial losses and economic insecurity; emotional exhaustion and overall nervousness; mistrust in directives from government and health authorities; and inner conflicts about complying with confinement orders, resulting in feelings of anger or helplessness. In particular, the possible exposure to the illness may trigger insomnia and PTSD symptoms. (Pfefferbaum and North 2020; Rajkumar 2020).

Clinical reports show that a new traumatic event, or an extremely stressful situation, is likely to stimulate earlier traumatic memories and trigger prior anxieties and their reenactment (Briere and Scott 2015). Indeed, our clinical observations show that for individuals in psychotherapy prior to the eruption of the pandemic, the encounter with the new stressful situation is not only likely to aggravate their previously acknowledged psychological distress, but also to stimulate the emergence of past painful experiences that did not surface earlier in therapy. The disruption of the therapeutic setting and having to change to using teletherapy may symbolize for clients their loss of control over their private lives, and often requires facing their fear of technology, dealing with new aspects of self-exposure and privacy, and coping with an unfamiliar relational experience. Our collected accounts from professionals show that, on the concrete level, clients' financial worries about the affordability of psychotherapy and their reluctance from teletherapy emerged as major obstacles to continuing and sustaining the therapy.

\section{Therapist's Experiences of Change}

The pandemic induces numerous personal and professional challenges for therapists. As it presents global health risks and forces demands for life routine changes for all, the pandemic creates a "shared traumatic reality," involving both therapists and clients. This concept relates to a dangerous or traumatic situation in which both therapists and clients are exposed to the same collective threat or disaster. In discussing insights from therapy work in the aftermath of the trauma of 9/11 in New York City, Saakvitne (2002) highlights both the benefits and vulnerabilities experienced by therapists in a shared traumatic reality. She observes that working under such conditions may contribute to therapists' sense of agency, lead to creativity in arranging modified therapeutic settings, and provide feelings of fulfillment in helping clients. However, she points to the potential perils of emotional strains and especially the emotional risk of "vicarious traumatization," resulting from the therapists' exposure to the distress of their clients, while facing their own emotional challenges.

Cohen et al. (2014) have identified similar benefits and risks associated with a shared traumatic reality for therapists, working with children and their caregivers in the aftermath of war in Israel. While therapists exhibited feelings of dedication, agency and satisfaction in their work, many reported suffering from various PTSD symptoms. Their emotional overload contributed at times to a blurring of boundaries in therapy; to the emergence of a defensive avoidance of trauma symbols expressed by the treated children; and to difficulties in applying their previously acquired traumafocused therapeutic techniques.

Attempts to contain both their own experience and the experiences of the clients may also result in the therapist's struggle with competing needs of self and others, leading to feelings of guilt and shame for favoring either set of needs (Baum 2012; Saakvitne 2002). Indeed, we gathered from our colleagues that Covid-19 mandated quarantine, lead therapists to feel conflicted between responsibilities towards clients and their personal and familial responsibilities.

\section{Changes in Therapeutic Relationships}

The shared experiences of the Covid-19 pandemic are likely to enhance a sense of symmetry between therapists and clients, changing the asymmetry that typically defines therapeutic relationships. Previous reports of therapy during shared traumatic events, such as the New Orleans hurricane Katrina, require changes in traditional "rules" of conducting therapy, which in turn may evoke guilt and shame in the therapists (Boulanger 2013). The greater symmetry between therapist and client, attempting to cope with similar challenges, may change their mutual emotional availability for therapy. When these needs complement each other, i.e., a client's recognition of his/her need for the therapist and the therapist's wish to continue working and help the client, teletherapy can be arranged through flexible negotiations of time and medium. However, when conflictual needs of the therapist and the client become evident, such as limited psychological or time availability of the therapist or the client, feelings of disappointment and helplessness may ensue. The expectations often held by therapists, and/or their clients, that the therapist be "stronger, wiser and kinder," to use Bowlby's (1988) characterization of a good attachment figure, often need to be compromised.

Such situations require negotiations between therapists and clients about the resumption and the new set-up of the therapy that can lead, in certain instances, to accepting temporary withholding of the therapy, while still maintaining an informal caring connection. These negotiations can also result in the therapists making thoughtful adaptations to the therapy. Such adaptations may include altering the frequency of sessions, modifying fees for financially distressed clients, and agreeing to experiment with the use of various modalities of teletherapy, in attempts to overcome the initial reluctance from therapy online. The success of such negotiations 
may depend on the conscious attempt of the therapist to regulate his/her emotions through various individual practices, such as mindfulness and self-compassion (Bibeau et al. 2015; Bourgault and Dionne 2019), and the use of professional resources, such as supervision and group support.

\section{Moving to Teletherapy}

In the past decade, the amount of online psychotherapy has gradually increased. Explanations for this phenomenon include the development of information technologies, transitioning to online platforms for various convenience needs, and attempts to offer psychotherapy in distant and rural areas (Wind et al. 2020). Several studies demonstrate the potential of online therapy in preserving the therapeutic alliance and their efficacy in improving clients' depression, stress, and PTSD (Spijkerman et al. 2016; Turgoose et al. 2017). However, some evidence points to slightly less effectiveness in online therapy in comparison to in-person therapy (Gordon et al. 2015).

Covid-19 forced a universal, sharp, transition of psychotherapists to using online working modes, requiring them to adapt and overcome difficulties related to such formats. Past reviews have identified impediments for clients and therapists using teletherapy. These include feelings of anxiety related to the technological world, difficulty in creating a private place to conduct sessions, and concerns related both to confidentiality and to unintended self-exposure (Gordon et al. 2015; Turgoose et al. 2017). Therapists themselves may feel unsettled by the forced abandonment of habitual means of conducting therapy, anxious about exposing their technological limitations, and insecure about their capacity to do competent work under such circumstances. Pronouncedly, they may be apprehensive about the loss of intimacy and emotional connection through a "cool medium" (à la Marshall McLuhan) that provides limited sensory data and deprives participants of a sense of full physical presence and tangible energy.

We suggest that to counter these potential limitations of teletherapy, and to concomitantly respond to the emergent stresses, therapists need to access and consolidate knowledge of psychotherapeutic principles and techniques that amplify the relational and experiential aspects of the therapy process. Recent research supports the clinical premise that in the context of a safe therapeutic relationship, past painful experiences, often defended-against, can be accessed, processed, and bring-about positive change. In particular, exploring trauma memories allows the individual to construct new meaning as well as to process maladaptive emotions associated with confusion and traumatic events, such as guilt and shame (Khayyat-Abuaita et al. 2019). Furthermore, it is recognized that a well-established therapeutic relationship is key for achieving results in all kinds of trauma-focused work and that attention to experiential principles, mainly emotion processing and emotion regulation, is essential to the successful progression of the therapeutic process (Murphy et al. 2019).

The Accelerated Experiential Dynamic Psychotherapy (AEDP) model may compatibly serve therapists in overcoming the current challenges, due to its unique combination of experiential, relational, and psychodynamic elements. Particularly relevant for these times is the aiming for inner emotional processing, while reducing attention to concrete temporary external conditions (Fosha 2001). The approach offers clinical interventions that may be relevant for therapists from varying therapeutic traditions.

\section{Adapting a model for teletherapy inspired by AEDP}

Based on attachment theory (Bowlby 1988), the AEDP model emphasizes the compassionate understanding of clients with their defensive internal working model, and the importance of creating security in the therapeutic relationship. Additionally, relying on therapeutic assumptions regarding trauma treatment and emotions research, the therapist encourages the client to be in contact with his/ her emotional experiences, as the main means for creating change (Fosha 2000). Adopting the premise that emotional experiences lie within bodily experiences and can be explicitly verbalized through various ways of expression (Van Der Kolk 2014), the therapist uses therapeutic interventions that cultivate awareness to emerging emotions, bodily sensations and images.

AEDP proposes a therapeutic process intended to create affective changes through being in contact with core emotions within a safely attached relationship. Core emotions are categorical and universal, and serve as important components, both for survival and for adaptation. Core emotions such as fear, anger and sadness, may be avoided during stressful times, as a defensive solution against a threat to attachment and adaptation needs (Fosha 2001). These defenses take a toll on one's ability to build satisfactory relationships and a stable sense of self.

The aim in therapy is to create the conditions of actually feeling experiences in vivo, in the safety of a securely attached therapeutic relationship. Feeling immersed in these experiences and processing them with reduced defensiveness, thanks to the support of the therapist, can lead to the emergence of new perspectives and understandings related to oneself and the situation. These are re-connected to new, more adaptable emotions, such as sorrow, acceptance, and self-appreciation. At the end of a session, the therapeutic work is reviewed and meta-processed. The meta-processing aids the client to become aware of the experienced 
transformation, express new realizations and self-reflections, and experience healing affects related to recovery, growth and resilience (Fosha 2013).

Keeping in mind the Covid-19 challenges to therapy described earlier, we introduce the following considerations borrowed from AEDP that seem most relevant to three main interrelated aspects of the teletherapy: the stance of the therapist, the notion of being in "the heart and mind" of the therapist, and reflective relational interventions.

\section{The Therapist's Stance}

Assuming a parental-like role, the therapist's stance in the dyadic therapeutic relationship invites corrective relational experiences. The therapist contains the client's experience and views the client "at his best" even if the client finds it difficult to see himself in such a positive light. The therapist attends to the client's defenses with compassion and moves to soften them through the continuous affirmation of the goodness, strength, and courage of the client. He/ she expresses delight in their client by identifying the client's healthy strivings and highlighting them. These received affirmations may empower the client, facilitate inner exploration, and improve one's sense of self (Fosha 2005).

Given the changes in the therapist due to Covid-19 experiences, it is important for the therapist to keep in mind that his/her feelings and stance toward the client and his/ her experiences do not need to be affected by the move to teletherapy. However, it is important to consider that the client's new experiences may change his/her outlook on the therapeutic relationship and process. Therefore, the therapist may need to re-affirm emphatically his/her positive regard for the client, and to regenerate the familiarity of their former relationship. This can be accomplished, first within the therapist himself, through experiential means such as recalling the sensations and feelings in past interactions with the client and imagining positive aspects of their interaction. Subsequently, the therapist can become teletherapeutic by conveying this accessed familiar experience to the client, so that both can recapture their emotional closeness, despite the lack of the shared physical space.

\section{Client's Felt Sense of Being "in the Heart and Mind of the Therapist"}

The client's sense of self involves a relational aspect, associated with his/her sense of presence and impact within the dyadic therapeutic relationship. A client who internalizes the belief that his/her well-being is held in the heart and mind of the therapist, experiences agency by becoming aware of the influence of his/her mere existence on the therapist's emotional life (Fosha 2009). The current circumstances call for creating opportunities to highlight this sense of the client of being responded to within the intersubjective experience of therapy. Examples include accommodating the therapeutic settings to the needs of the client when possible, expressing care for the health of the client and his/her close ones, and the remembering of personal details in the client's history that may be of relevance to the situation.

\section{Interventions to Enhance Teletherapy}

\section{Promoting Experiential Work}

With the intention of conducting a process that is experientially focused, the therapist pays close attention to the emotions experienced by the client, moment by moment, rather than focusing on content. The client's internal process is assumed to progress with the mutual adherence and attentiveness of both client and therapist to the client's emotional experience (Fosha 2001). The therapist may choose to use material emerging for the client through any association or trigger, as an opportunity to approach a meaningful emotional process. The client's sharing of experiences related to the Covid-19 situation, or feelings about "disturbances" to the ongoing teletherapy, may serve as opportunities to access themes of deep emotional and psychological meanings. Through the therapist's encouragement of the client to stay with emotions as they emerge and the emotional accompaniment of the therapist, associations with earlier past hurtful or traumatic experiences may surface, and various themes may be tapped, such as safety, loss, attachment wounds, defenses, and sense of self. A short example of this therapeutic process is presented below.

\section{Tracking Moment-by-Moment}

In teletherapy, therapists, together with their clients, need to consider and settle on a medium that would allow them setting conditions similar, as much as possible, to essential ones provided by conventional therapy. These involve uninterrupted private communication and clarity of the possible verbal and non-verbal exchanges. This may include paying attention and adjusting volume, voice clarity or the positioning of the camera.

Aiming to elicit the client's experience with precision, the therapist tries to closely track the client's verbal and nonverbal expressions evident in his/her face and body. In using teletherapy, therapists can track the client's voice, facial expressions, the upper body area, and movement. In teletherapy these limited cues may be closer in view, or more directly audible in comparison to face to face therapy. However, the therapist needs to become proficient in noticing 
changes in tone and prosody, subtle facial movements and changes in expressions, reflecting sensations and feelings. These and additional cues from the client's breath and posture also reflect the client's general feelings of safety and may contribute to the degree of physiological and emotional synchronization and reciprocity that take place in dyadic interactions (Porges 2003). The therapist's checking with the client the accuracy of his/her interpretations of these cues contributes to their mutual work and creates for the client a positive sense of his/her experience being accurately perceived, understood, and verbalized in a compassionate manner. In a parallel manner, the therapist needs to become cognizant of the clarity and intensity of his/her own communications to the client, emphasizing emotional involvement and reactions by heightened nonverbal and para-verbal means.

\section{Dyadic Regulation}

Internal capacities for regulation of emotions are essential in accomplishing deep emotional therapeutic work and restoring resilience. Using an intersubjective approach, dyadic regulation contributes to the client's regulation, as the therapist and client progress with mutual coordination affectively and experientially. Working via teletherapy during a stressful time, the therapist may need to focus on helping the client with self-regulation. Regulation techniques that may prove to be useful include the therapist's slowing down speech, expressing care and compassion, leading in joint breathing exercises, using grounding techniques, or helping the clients boost their energy level through guided bodily movements (Prenn, 2011; Van Der Kolk 2014).

\section{Relational Considerations and Techniques}

Various therapeutic models focus their attention on the relational elements of treatment and to the quality of the relationship, especially in working with trauma (Murphy et al. 2019). Considering the traumatic aspects of these times, we suggest techniques for addressing relational aspects in psychotherapy while working online. These include ways of undoing the client's "aloneness" (sense of being alone with their experience), promoting emotional engagement, and encouraging reflections on the therapeutic relationship and process.

\section{Undoing Aloneness}

A key premise of AEDP is the assumption that trauma develops in a relational context when the person goes through a profound negative or bewildering experience, while experiencing a sense of aloneness. Therefore, a therapeutic relationship which cultivates in therapy the client's profound experience of feeling belonged and cared for, has a crucial impact on his/her sense of safety and sense of self (Fosha 2009). The notion of aloneness is specifically relevant in the Covid-19 era, as clients experience survival anxieties and general insecurities and uncertainty, while concurrently being distant and isolated from their support networks. In teletherapy, the risk of loneliness implies the need to emphasize and communicate the safety of the securely attached therapeutic relationship. Verbal and nonverbal messages may act as reassurance of the therapist's presence, such as, repeating empathically the client's verbal expressions, reflecting observed feelings, and actively reminding the client of his/her presence when strong feelings emerge. Instances of subtle yet meaningful messages to undo the client's aloneness may include the therapist's referring to them both in plural ("we... let's..."), and explicitly encouraging the client to emotionally include the therapist in his experience ("stay with what comes up and stay with me"). Nonverbal interventions that enhance the client's feelings of togetherness consist of cues, such as, maintaining eye contact and waiting with kind eyes and a compassionate look, even when the client is looking away.

\section{The Therapist's Emotional Engagement}

In teletherapy, the therapist's emotional engagement may act as a useful relational tool in preserving the therapeutic atmosphere. The therapist's being explicit about his/her personal emotional experiences and using self-exposure in relation to the ways in which he/she is impacted by the dyadic relationship and the client's process, are expected to contribute to the forming of a joint process of shared experiences, and to the client's progression towards healing (Prenn 2011). The mutual sharing of experiences may buffer the feelings of limited intimacy in teletherapy. During unstable times, such as Covid-19, this sharing contributes to the building of a stable intimate world, which both the client and therapist belong to, promoting more endurance and resilience. The effect of the therapist's clear expression of her emotional engagement on the progression of the therapeutic process may become evident in the forthcoming section presenting the clinical case.

\section{Reflections on the Therapeutic Relationship and Process}

Using a relational framework, the therapist often invites the client to share explicit reflections on their therapeutic relationship. Through this tool, the therapist and client are able to stay connected to their experience of authentic dyadic interaction, enhance secure attachment, undo feelings of aloneness, and promote inner exploration (Fosha 2013). In teletherapy, the therapist may need to check with the client more frequently how they feel vis-a-vis the therapist at 
certain moments, how they feel with the process of sharing and with the therapy work, especially in view of the differences in the medium and the psychological impact of the pandemic. The therapist might suggest "Let's take a moment; I wonder, as you look back at our work, what feelings or sensations surface for you?" The therapist can encourage authentic sharing through reassuring statements such as: "whatever comes up for you would be good for us." When the client expresses his/her experience, the therapist may deepen this line of exploration asking about feelings, bodily sensations, and reflective thoughts regarding the process. For instance, "and how is that for you to feel this here today, in comparison to back then" and "how is it to feel this together, here, with me?".

Through the therapist's emotional engagement and the reflective questions, the process of enticing the client to being emotionally open to receive the therapist's emotional inputs and to authentically share their own interactive experiences, may contribute to the client's emotional flexibility and ability to integrate his/her experiences with those of another.

The following account of the therapist of a teletherapy session with a client illustrates the discussed principles, applied in practice.

\section{Teletherapy in the Case of $\mathrm{K}$}

Following the official public order to adhere to social distancing, I moved to work via teletherapy, but $\mathrm{K}$ felt reluctant to work this way. After hesitations, when she agreed to try online therapy, I felt appreciation for her effort and courage, and looked forward to continuing our connection. As we started the session, $\mathrm{K}$ appeared uneasy about viewing herself on the screen while talking. She realized that the platform allowed seeing both participants on the screen or hiding either image. K began switching the "on" and "off" tabs, debating and looking at herself with an irritated expression. I felt her rising tension and validated her confusion. I suggested we stay with her experience. Rather than trying to address solely the technical solution of viewing her image on the screen, we experientially began working on regulation: As I attentively kept a kind eye contact with her and used a slow and calming voice, I felt that these interventions gradually reminded her of "us." I suddenly felt it strongly through seeing her changed facial expression and directness of her gaze at me. I also felt it through my own experience: through my intersubjective re-emerging experience of our relationship, recollecting the way she usually experiences me. This feeling of known mutual resonance enabled me to be present 'in the moment' and attend to our work as if we were not using screens: I mentioned my sudden feelings of relief and joy in emotionally reconnecting (me: "Ah, K,
I can now feel 'us' again, just as before, what a relief, I am so glad!”). K giggled briefly and I joined her giggle. K, amused, mentioned that now all we need is the background of my office, behind my image. I smiled, agreed, and thought aloud about pasting such a picture behind both of us.

Now, it felt as though we were back, riding the process together, with trust and awareness of our connection. We could start working on the content that emerged, rather than dealing with the technical issue of turning off her image from the screen. We focused on the emotional and relational aspects of viewing herself. $\mathrm{K}$ responded by recounting an incident from her childhood, when her sibling commented on how odd she looked and behaved. This memory triggered additional memories of feeling criticized and rejected by her family, feeling very much alone, a feeling that lingers on to this day. I accompanied $\mathrm{K}$ in experientially going back in time, and to access her preciously avoided related core emotions. This allowed feelings of grief to surface and be safely shared.

At one point in the session, she had to go out to another room to get a new package of tissues. That moment felt like a step out of her "inner world" and from the close dyadic interaction. When she returned, I felt the urge to act and help us quickly reestablish the closeness we had achieved and the safety for continued inner exploration. I mentioned a humorous comment, K giggled, I giggled back-and we started to reconnect. I then took a deep breath, searched for eye contact with her, then took another breath, which she joined with me (showing our dyadic regulation), and we were back to work.

As I was looking at her, expressing compassion and affirmation, she accessed her yearnings for what she would like to feel about herself. She was able to experience with my emotional involvement and support "mourning-of-the-self," deep sorrow for her unacknowledged and long hidden hurt. This served as motivational striving, which I validated, longing to sense herself as "a person who deserves appreciation." Though the process of mourning and deep sadness was evident, these experiences were justifiably emerging from a position of self-acknowledgement. This saddened core emotion created an uplifting feeling of a healthy response, of speaking on behalf of herself and the consolidated wish to feel her self-worth. I shared with her my appreciation and delight in her new state and process of transformation.

\section{Integration of Theory and Practice}

This described session with " $\mathrm{K}$ " demonstrates interventions that enhance movement towards both client's and therapist's emotional engagement, tapping into inner experiences while being in a safely attached relationship. Enhancing the client's regulation through compassion, eye contact and dyadic regulated breathing, enables the client to focus adaptively 
on her inner experience. The therapist, showing affirmative regard towards the client, being engaged and delivering a sense of containment in mind and heart, creates a path for undoing the client's aloneness. These interventions, applicable via teletherapy, promote safe attachment, courageous exploration and emergence of natural motivational strivings, resulting in self-compassion and resilience. We present the matching of the presented interventions for the challenges of Covid 19 as a schematic dynamic model illustrated in Fig. 1.

In Fig. 1 the solid line boxes present the challenges to the therapeutic relationship, as a result of the Covid-19 pandemic, related experiences of change in clients and therapists, and the implications of the adjustment to the modality to teletherapy. The aforementioned supporting interventions for addressing these challenges are alluded to in the dashed line boxes. The therapist's regenerating the caring feelings and adapting the familiar stance towards the client, noted in box (a), aim to deflate any apprehensions or negative feelings towards the therapy, stemming from the therapist's recent personal circumstances. This stance of the therapist serves as a corrective measure for possible negative effects of the client's personal experience of change. Through additional awareness of his/her previous intimate involvement with the client, the therapist communicates to the client that he/she is holding the client in mind and heart (marked in box b). This chain of influence positively affects the re-establishment of the sense of their reciprocal relationship, which may have been at risk due to the changed circumstances. The therapist's maintaining the role of a significant attachment figure involves the emergence of corrective emotional and relational experiences. These may directly enable the client to loosen her/his defenses and to follow the therapist's invitations to explore emotional experiences and connect to their core feelings.

The suggested intervention tools (noted in box c) help the client progress through such a therapeutic process. They aim to help clients to self-regulate, encourage them to draw their attention to inner experiences and promote the explicit expression of these experiences. Furthermore, these interventions that revolve around the client's experience, present the client with a safe and empowering sense of leading the process themselves, while the therapist follows their lead, with curiosity, engagement, and emotional self-exposure, in reciprocal coordination and resonance.

The client's tasks in the therapeutic process (noted in box d) involve paying attention to her/his safety and cultivating a

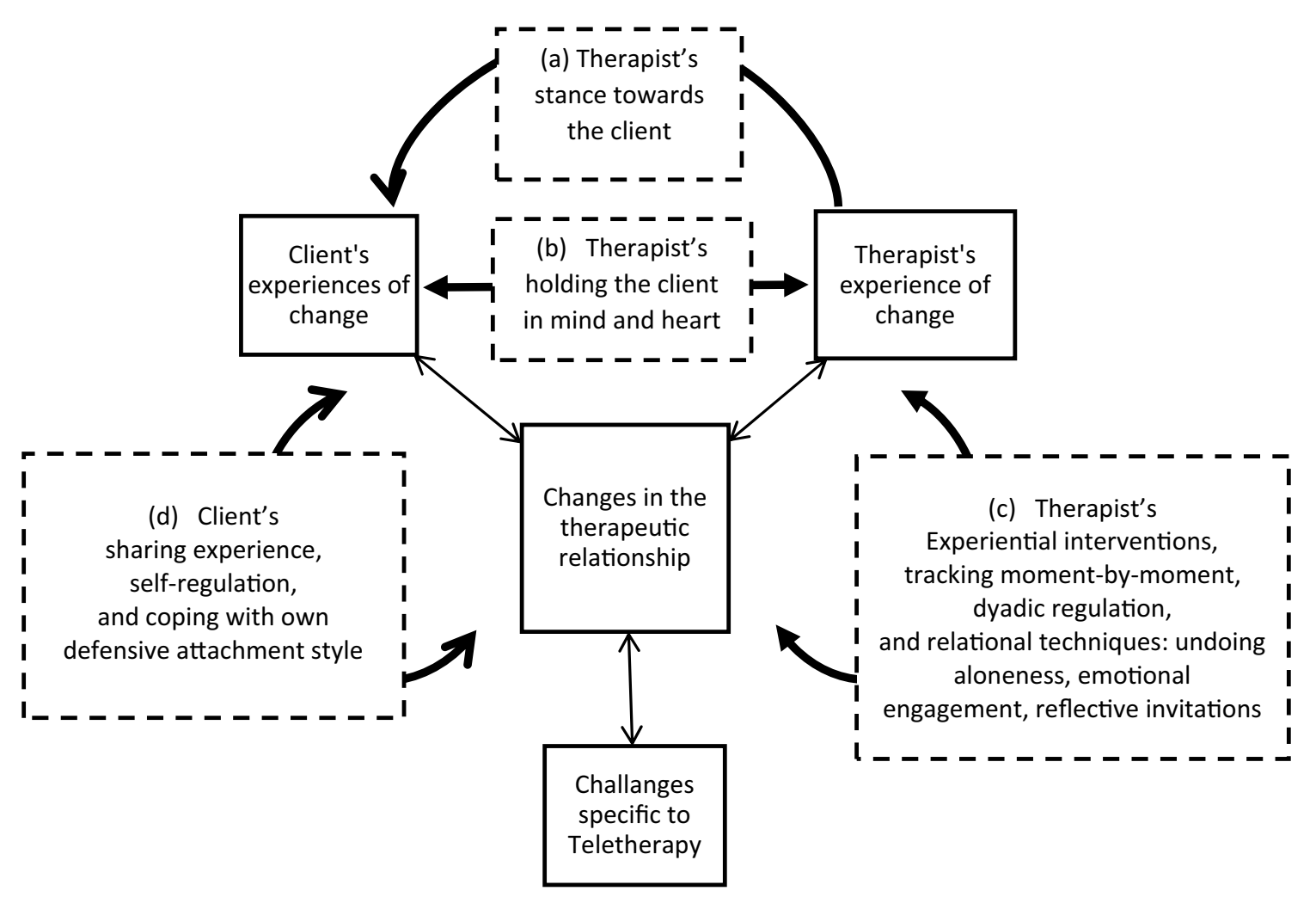

Boxes marked by solid lines denote new challenges due to the pandemic;

Boxes marked by dashed lines include suggested tools to promote teletherapy

Fig. 1 The use of AEDP tools to promote client's change processes during Covid-19 
sense of self within the dyadic relationship, enlisting motivational strivings towards transformation and wellbeing. The therapist's interventions, both those directed at him/herself as maintaining a therapeutic stance towards the client (boxes $a$ and b) and those explicitly directed towards the client (c), together with the tasks undertaken by the client, work in synergy, resulting in a positively changed therapeutic relationship and a beneficial healing process for the client.

\section{Conclusions}

In reviewing existing past and recent reports, and by examining the present accounts of our supervisees and colleagues, we identify some major challenges to the therapeutic work, related to the particular situation of the Covid-19 era. We focus on essential therapeutic principles and interventions designed to foster safely attached relationships, especially needed at this time. This effort is considered a pre-condition for meaningful experiential transformative work. We demonstrate how to use AEDP techniques for supporting the emergence of emotions, processing them, and incorporating deep understandings about the self, connected to present and past relational experiences.

Considering the future, we may unfortunately expect a growing need for mental health services and an increase in the use of teletherapy (Wind et al. 2020). The mindful adoption of experiential and relational tools by therapists, using various models of clinical work, may help them become more teletherapeutic and overcome the potential limitations of online psychotherapy. Nevertheless, it is natural for therapists and clients to miss or long for the actual physical presence of each other. Sharing the sense of loss and mourning the changes, may lead both therapist and client to appreciate their past mutual experiences and the uniqueness and resilience of their bond, thus restoring safety. A different relational experience may develop when a therapy process begins online, without prior physical acquaintance. Clients who choose to enter teletherapy are usually more accepting of the medium and comfortable with it. In these cases, the therapist needs to invest in creating a sense of closeness that would generate feelings of togetherness and familiarity, in an accelerated fashion, using many of the techniques mentioned earlier. Often more attention needs to be devoted in such cases to discussing the rules of the setting in order to ascertain stability, continuity, and privacy.

Teletherapy with individuals in the Covid-19 era often takes place in home settings, and the nearby presence of family members cannot be ignored, physically or psychologically. This particular condition may require integrating more systemic approaches and transitioning to additional forms of dyadic and familial interventions. Thus, the new challenges of this era necessitate further conceptual attempts at theory integration and modification of techniques, accompanied by clinical-related research. Moreover, we believe that much more attention, beyond the scope of this paper, should be devoted to highly vulnerable individuals, such as those physically affected by the disease, suffering loss of loved-ones due to the pandemic, and frontline health-care workers (Inchausti et al. 2020).

In addition to the emotional toll of the shared traumatic reality, working in teletherapy requires concentration and may prove more demanding than face-to-face therapy. Implementing self-care practices and seeking support from colleagues may be essential for the wellbeing of therapists and the quality of work (Norcross 2000).

Author Contributions Both authors contributed to the paper in the conception and design, in material preparation and writing. The drafts of the paper have been written by both authors, with cooperation. each of them expanding in their field of expertise, AEDP and traumatic shared experiences. All authors read and approved the final manuscript.

Funding Not applicable.

\section{Complaince with Ethical Standards}

Conflicts of interest We have no conflict of interest.

Code of Ethics In order to comply with the code of ethics, we have used de-identification criterion to present a clinical case. This criterion is used with the material under the title "The case of K". The presentation includes anonymous information that does not identify a certain individual.

\section{References}

Asmundson, J. G., \& Taylor, S. (2020). Coronaphobia: Fear and the 2019-nCoV outbreak. Journal of Anxiety Disorders, 70, 102196. https://doi.org/10.1016/j.janxdis.2020.102196.

Baum, N. (2012). Trap of conflicting needs: Helping professionals in the wake of a shared traumatic reality. Clinical Social Work Journal, 40(1), 37-45. https://doi.org/10.1007/s10615-011-0347-0.

Bibeau, M., Dionne, F., \& Leblanc, J. (2015). Can compassion meditation contribute to the development of psychotherapists' empathy? A review. Mindfulness, 7(1), 255-263. https://doi.org/10.1007/ s12671-015-0439-y.

Boulanger, G. (2013). Fearful symmetry: Shared trauma in New Orleans after Hurricane Katrina. Psychoanalytic Dialogues, 23(1), 31-44. https://doi.org/10.1080/10481885.2013.752700.

Bourgault, M., \& Dionne, F. (2019). Therapeutic presence and mindfulness: Mediating role of self-compassion and psychological distress among psychologists. Mindfulness, 10(4), 650-656. https:// doi.org/10.1007/s12671-018-1015-z.

Bowlby, J. (1988). A secure base: Parent-child attachment and healthy human development. New York, NY: Basic Books.

Briere, J., \& Scott, C. (2015). Complex trauma in adolescents and adults: Effects and treatment. Psychiatric Clinics of North America, 38, 515-527. https://doi.org/10.1016/j.psc.2015.05.004.

Brooks, S. K., Webster, R. K., Smith, L. E., Woodland, L., Wessely, S., Greenberg, N., et al. (2020). The psychological impact of 
quarantine and how to reduce it: Rapid review of the evidence. The Lancet, 395, 912-920. https://doi.org/10.1016/S0140 $-6736(20) 30460-8$.

Cohen, E., Roer-Strier, D., Menachem, M., Fingher-Amitai, S., \& Israeli, N. (2014). "Common-Fate": Therapists benefits and perils in conducting child therapy following the shared traumatic reality of war. Clinical Social Work Journal, 43(1), 77-88. https://doi. org/10.1007/s10615-014-0499-9.

Fosha, D. (2000). The transforming power of affect: A model for accelerated change. New York, NY: Basic Books.

Fosha, D. (2001). The dyadic regulation of affect. Journal of Clinical Psychology, 57(2), 227-242. https://doi.org/10.1002/10974679(200102)57:2<227:AID-JCLP8>3.0.CO;2-1.

Fosha, D. (2005). Emotion, true self, true other, core state: Toward a clinical theory of affective change process. Psychoanalytic Review, 94(4), 513-552. https://doi.org/10.1521/prev.2005.92.4.513.

Fosha, D. (2009). Healing attachment trauma with attachment (and then some!). In M. Kerman (Ed.), Clinical pearls of wisdom: 21 leading therapists offer their key insights (pp. 43-56). New York: Norton.

Fosha, D. (2013). Emotion and recognition at work: Energy, vitality, pleasure, truth, desire \& the emergent phenomenology of transformational experience. The Neuropsychotherapist, 2, 28-51.

Gordon, R. M., Wang, X., \& Tune, J. (2015). Comparing psychodynamic teaching, supervision, and psychotherapy over videoconferencing technology with Chinese students. Psychodynamic Psychiatry, 43(4), 585-599. https://doi.org/10.1521/pdps.2015.43.4.585.

Inchausti, F., MacBeth, A., Hasson-Ihayon, I., \& Dimaggio, G. (2020). Psychological intervention and covid-19: What we know so far and what we can do. Journal of Contemporary Psychotherapy. https://doi.org/10.1007/s10879-020-09460-w.

Khayyat-Abuaita, U., Paivio, S., Pascual-Leone, A., \& Harrington, S. (2019). Emotional processing of trauma narratives is a predictor of outcome in emotion-focused therapy for complex trauma. Psychotherapy, 56(4), 526-536. https://doi.org/10.1037/pst0000238.

Murphy, D., Elliott, R., \& Carrick, L. (2019). Identifying and developing therapeutic principles for trauma-focused work in personcentered and emotion-focused therapies. Counseling and Psychotherapy Research. https://doi.org/10.1002/capr.12235.
Norcross, J. C. (2000). Psychotherapist self-care: Practitionertested, research-informed strategies. Professional Psychology: Research and Practice, 31(6), 710-713. https://doi. org/10.1037/0735-7028.31.6.710.

Pfefferbaum, B., \& North, C. S. (2020). Mental health and the Covid19 pandemic. New England Journal of Medicine, 382(20), 1-3. https://doi.org/10.1056/NEJMp2008017.

Porges, S. W. (2003). Social engagement and attachment: A phylogenetic perspective. Annals of New York Academy of Science, 1008, 31-47. https://doi.org/10.1196/annals.1301.004.

Prenn, N. (2011). Mind the gap: AEDP interventions translating attachment theory into clinical practice. Journal of Psychotherapy Integration, 21(3), 308-329. https://doi.org/10.1037/a0025491.

Rajkumar, R. P. (2020). COVID-19 and mental health: A review of the existing literature. Asian Journal of Psychiatry, 52, 1-5. https:// doi.org/10.1016/j.ajp.2020.102066.

Saakvitne, K. W. (2002). Shared trauma: The therapist's increased vulnerability. Psychoanalytic Dialogues, 12(3), 443-449. https://doi. org/10.1080/10481881209348678.

Spijkerman, M. P. J., Pots, W. T. M., \& Bohlmeijer, E. T. (2016). Effectiveness of online mindfulness-based interventions in improving mental health: A review and meta-analysis of randomized controlled trials. Clinical Psychology Review, 45, 102-114. https:// doi.org/10.1016/j.cpr.2016.03.009.

Turgoose, D., Ashwick, R., \& Murphy, D. (2017). Systemic review of lessons learned from delivering tele-therapy to veterans with posttraumatic stress disorder. Journal of Telemedicine and Telecare, 24(9), 575-585. https://doi.org/10.1177/1357633X17730443.

Van Der Kolk, B. (2014). The body keeps the score. New York: Viking.

Wind, T. R., Rijkeboer, M., Andersson, G., \& Riper, H. (2020). The COVID-19 pandemic: The 'black swan' for mental health care and a turning point for e-health. Internet Interventions, 20, 100317. https://doi.org/10.1016/j.invent.2020.100317.

Publisher's Note Springer Nature remains neutral with regard to jurisdictional claims in published maps and institutional affiliations. 